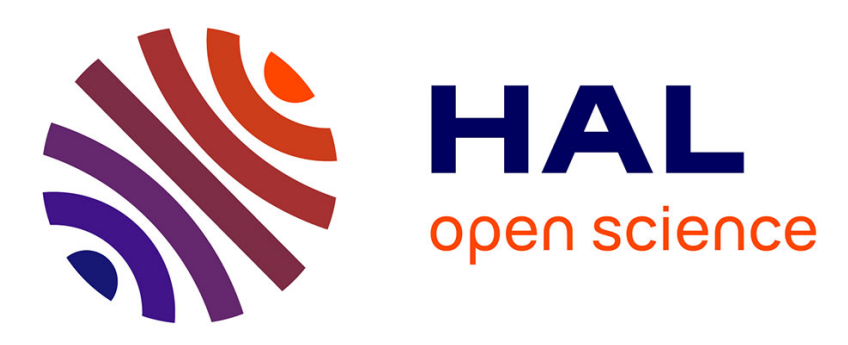

\title{
Passive coherent combination of two ultrafast rod type fiber chirped pulse amplifiers
}

\author{
Yoann Zaouter, Louis Daniault, Marc Hanna, Dimitris N. Papadopoulos, \\ Franck Morin, Clemens Hönninger, Frédéric Druon, Eric Mottay, Patrick
}

Georges

\section{To cite this version:}

Yoann Zaouter, Louis Daniault, Marc Hanna, Dimitris N. Papadopoulos, Franck Morin, et al.. Passive coherent combination of two ultrafast rod type fiber chirped pulse amplifiers. Optics Letters, 2012, pp.1460. hal-00702173

\section{HAL Id: hal-00702173 \\ https://hal-iogs.archives-ouvertes.fr/hal-00702173}

Submitted on 29 May 2012

HAL is a multi-disciplinary open access archive for the deposit and dissemination of scientific research documents, whether they are published or not. The documents may come from teaching and research institutions in France or abroad, or from public or private research centers.
L'archive ouverte pluridisciplinaire HAL, est destinée au dépôt et à la diffusion de documents scientifiques de niveau recherche, publiés ou non, émanant des établissements d'enseignement et de recherche français ou étrangers, des laboratoires publics ou privés. 


\title{
Passive coherent combination of two ultrafast rod type fiber chirped pulse amplifiers
}

\author{
Yoann Zaouter, ${ }^{1, *}$ Louis Daniault, ${ }^{2}$ Marc Hanna ${ }^{2}$, Dimitris N. Papadopoulos ${ }^{3}$, Franck Morin ${ }^{1}$, \\ Clemens Hönninger ${ }^{1}$, Eric Mottay $^{1}$, Frédéric Druon $^{2}$, and Patrick Georges ${ }^{2}$ \\ ${ }^{1}$ Amplitude Systemes, 11 avenue de Canteranne, Cité de la Photonique, 33600, Pessac, France \\ ${ }^{2}$ Laboratoire Charles Fabry, Institut d'Optique, CNRS, Université Paris-Sud, 2 av. Augustin Fresnel, 91127 Palaiseau Cedex, \\ France \\ ${ }^{3}$ Institut de la Lumière Extrême, CNRS, Ecole Polytechnique, ENSTA Paristech, Institut d'Optique, Université Paris Sud, \\ Palaiseau Cedex, France \\ *Corresponding author: yzaouter@amplitude-systemes.com
}

\author{
Received Month X, XXXX; revised Month X, XXXX; accepted Month X, \\ XXXX; posted Month X, XXXX (Doc. ID XXXXX); published Month X, XXXX
}

\begin{abstract}
Using passive coherent beam combining of two ultrafast fiber amplifiers, we demonstrate the generation of high temporal quality $300 \mathrm{fs}$ and $650 \mu \mathrm{J}$ pulses i.e. $60 \mathrm{~W}$ of average power at a repetition rate of $92 \mathrm{kHz}$. Furthermore at $2 \mathrm{MHz}$ of repetition rate a record coherent combining average power of $135 \mathrm{~W}$ before and $105 \mathrm{~W}$ after compression is measured. A combining efficiency higher than $90 \%$ is maintained over the whole range of output powers and repetition rates investigated demonstrating the efficiency and robustness of the passive combining technique. The measured pulse-to-pulse relative power fluctuation at high energy is $2 \%$, indicating that the system is essentially immune to environmental phase noise. We believe the passive combining method to be an attractive approach for compact multi-GW peak power femtosecond fiber-based sources.
\end{abstract}

OCIS Codes: $140.3615,140.3280,140.3380,060.2290$

The peak power obtained from high repetition rate ytterbium-doped ultrafast fiber amplifiers has grown extremely rapidly during the last decade to reach the multi-GW level owing to the development of very large mode area fiber designs [1]. To further scale this peak power and obtain compact and efficient high repetition rate high energy sources, the coherent combination of several such femtosecond fiber lasers has recently been demonstrated. Early demonstrations have involved an active control of the relative phase between two fiber chirped-pulse amplifiers (FCPA). A first experiment used the measurement of the polarization state of the combined beam, and a feedback on a piezo-mounted mirror located in one amplifying arm [2]. The same group scaled up the performances to a record energy of $3 \mathrm{~mJ}$ [3]. A second demonstration was based on of the frequency tagging technique [4], while the feedback is done through an integrated fiber-coupled $\mathrm{LiNbO}_{3}$ phase modulator [5]. Both these implementations have demonstrated feedback bandwidths in the $1-10 \mathrm{kHz}$ range. They require complex opto-electronic feedback schemes, and the stability of such sources in noisy environments will depend on the sophistication of the feedback loop.

Recently, we proposed an alternative technique to achieve coherent combining of two amplifiers in a passive architecture involving a Sagnac interferometer [6]. The phase stability of this simple scheme is inherently guaranteed by the fact that both combined beams travel along the exact same optical path in a counter propagating geometry. Our proof-of-principle experiment showed that the passive technique is compatible with high nonlinearity levels, and that the limiting factor is the ability to maintain the symmetry of both paths.

In this Letter, we use the passive combining architecture and rod-type fiber amplifiers to generate high temporal quality $300 \mathrm{fs}$ pulses with $650 \mu \mathrm{J}$ of energy per pulse at a repetition rate of $92 \mathrm{kHz}$. This corresponds to an average power of $60 \mathrm{~W}$, and a peak power in excess of $2 \mathrm{GW}$, demonstrating the potential of the technique in a high performance fiber system.

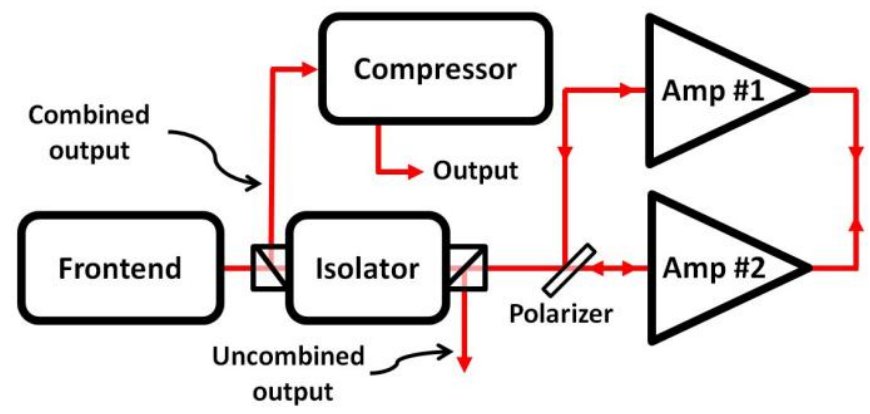

Figure 1: (Color online) Experimental setup

Figure 1 shows the experimental setup used in our work. To maintain a good temporal pulse quality simultaneously with a high energy level, we implement a moderately nonlinear FCPA in which the impact of nonlinearities is partially compensated by the dispersion mismatch of the stretcher and compressor units [7]. Furthermore, the two amplifiers that we intend to coherently combine are built within a Sagnac interferometer that is seeded, through an optical isolator, by a frontend. This frontend is composed of a passively mode-locked ultrafast oscillator, a pulse picker, a pulse stretcher, and a single-mode fiber pre-amplifier. The oscillator generates pulses at the central wavelength of $1030 \mathrm{~nm}$. The full width at half maximum of the spectrum is $9 \mathrm{~nm}$ and sustains pulse durations below 200 fs. The repetition rate of the oscillator of $\sim 25 \mathrm{MHz}$ is divided by an acousto-optic modulator pulse picker and can be varied 
from $92 \mathrm{kHz}$ to $25 \mathrm{MHz}$. Sufficient pulse stretching is then applied to the pulses in order to mitigate the impact of nonlinearities in the power amplifiers. The stretching ratio is designed to preserve a pulse duration of $\sim 600 \mathrm{ps}$ after the power amplifiers when operated at maximum gain. A single mode fiber amplifier is used to boost the average power to about $100 \mathrm{~mW}$, which is sufficient to seed both power amplifiers. An optical isolator is placed after the frontend to prevent any optical feedbacks coming from the power amplifiers. It is also used to separate the combined and the uncombined outputs of our passively coherently combined amplification scheme. The beam is split by a polarizer and each secondary beam seeds a power amplifier (denoted as Amp \#1 and Amp \#2, see Figure 1) made of state-of-the art ytterbium-doped rodtype photonic crystal fibers. These rigid fibers are kept straight in un-cooled V-groove holders and are optically pumped by $976 \mathrm{~nm}$ fiber coupled pump diodes. The mode field diameter of the fibers is $85 \mu \mathrm{m}$, the pump cladding diameter is $285 \mu \mathrm{m}$ and their length of $1 \mathrm{~m}$ ensures sufficient pump absorption and optical efficiency [8]. The outputs of each amplifier are connected to each other to close the Sagnac interferometer. Therefore, after the polarizer, the light propagating clockwise is first preamplified in Amp \#1 and then amplified in Amp \#2 while the light travelling counterclockwise is first pre-amplified in Amp \#2 before reaching its maximum power / energy at the output of Amp \#1. Both beams are then recombined on the polarizer, with linear polarizations orthogonal to each other. If both beams are in-phase, they form a combined output beam propagating back to the isolator with a linear polarization state at an angle of $45^{\circ}$. This beam is therefore transmitted back through the rotator and ejected by the polarizer located at the input of the isolator. The uncombined part of the beam is removed by the polarizer located at the output of the isolator. Measurement of the average power at both outputs allows us to compute the combination efficiency defined as the ratio between the combined power and the total power.

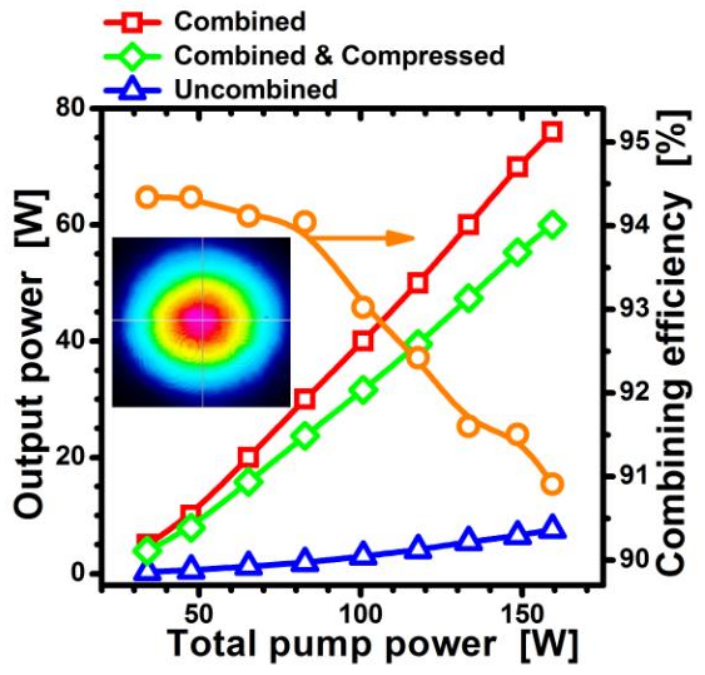

Figure 2: (Color online) Left scale: output powers of the amplifier as a function of pump power at a repetition rate of $92 \mathrm{kHz}$. Right scale: combining efficiency as a function of pump power. Inset: beam profile taken at maximum energy at the output of the system.
The path length for one round trip in the Sagnac interferometer is $5.5 \mathrm{~m}$, making this setup immune to all phase noises with frequency contents below $27 \mathrm{MHz}$. This large bandwidth essentially removes all environmental sources of noise such as acoustic and thermal fluctuations of the relative phase. To highlight the robustness of this architecture with respect to phase noise, we compare the pulse-to-pulse relative power fluctuation of the frontend to the one measured at the output at maximum energy. This pulse-to-pulse stability is $1.1 \%$ for the frontend, and reaches $2 \%$ at the maximum compressed energy. This very small decrease of the pulse-to-pulse stability for a nonlinear FCPA, built on an uncovered breadboard, clearly indicates that phase noise has very little impact, if any, on the global coherent combination stability. This is of particular importance if lasers using our passive coherent combination architecture are to be used in harsh environments or if the pulse-to-pulse stability is of particular importance for the targeted scientific or industrial applications. Finally, the coherently combined beam is sent through a compressor optimized to get the highest possible peak power. The high efficiency gratings used allow for only $21 \%$ loss in the compressor.

The passive coherent combination setup described above is first aligned at low power and low energy in order to obtain the best possible degree of combination related to spatial aspects such as beam profile and wavefront mismatch. We found out that slight differences in the rod type fibers end-facet preparation is currently the main limitation to the combination efficiency, with a maximum value of $\sim 95 \%$. The pulse picker is then set to downcounter the repetition rate of the frontend to $92 \mathrm{kHz}$. Figure 2 shows the amplifier characteristics at this repetition rate with the combined and uncombined average powers measured at the output ports of the isolator. At the maximum total pump power of $160 \mathrm{~W}$, we reach $75 \mathrm{~W}$ of passively coherently combined power, i.e. $815 \mu \mathrm{J}$, with a slope efficiency close to $60 \%$. It is noticeable that despite the accumulation of a large quantity of nonlinear phase shifts in the amplifiers (estimated to be $\sim 7 \mathrm{rad}$ per roundtrip), the combining efficiency decreases by less than $3.5 \%$. We investigated the origin of this loss and found out that nonlinearity mismatch between the two circulating directions is the main source of drop in combining efficiency. Indeed, at 75 $\mathrm{W}$ and $2 \mathrm{MHz}$ of repetition rate the rejected uncombined power is only $5 \mathrm{~W}$, resulting in a combining efficiency close to $94 \%$. At even higher average power we see another roll off in efficiency that originates from a worst spatial overlap of the two beams. Nevertheless, for $270 \mathrm{~W}$ of total pump power at $2 \mathrm{MHz}$ of repetition rate, we reached 135 $\mathrm{W}$ of coherently combined power with a passive coherent combining efficiency of $91.5 \%$. The compressed average power of $105 \mathrm{~W}$ is to our knowledge the highest average power ever generated by any active or passive coherently combined ultrashort amplifiers.

We now investigate the compressibility of the amplified pulses at maximum energy. At $92 \mathrm{kHz}$, the maximum average power measured after the compressor is $60 \mathrm{~W}$ (76 $\mathrm{W}$ before) i.e. $650 \mu \mathrm{J}$ of energy per pulse. Figure 2 shows the autocorrelation traces acquired with an intensity autocorrelator and independently measured with a 
second-harmonic generation frequency-resolved optical gating apparatus (FROG). These autocorrelations can hardly be differentiated and exhibit a FWHM duration of $400 \mathrm{fs}$. Figure 2 also shows the retrieved temporal profile of the pulse from the FROG measurement. Its duration is $300 \mathrm{fs}$, corresponding to a time-bandwitdh product (TBP) of 0.75 . The very good temporal quality obtained allows us to measure a peak power in excess of $2 \mathrm{GW}$.
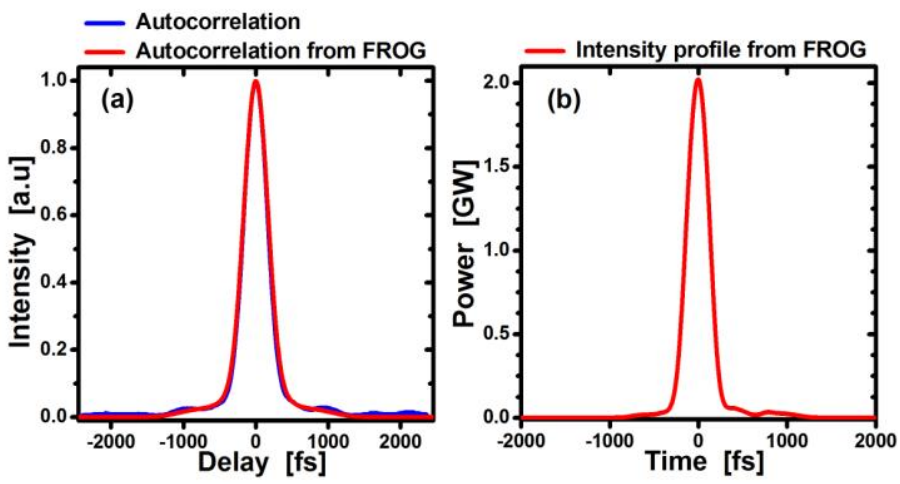

Figure 3: (Color online) (a) Autocorrelation traces measured at $650 \mu \mathrm{J}$ (blue) with an independent autocorrelator and (red) from a FROG measurement. (b) Retrieved temporal profile of the pulse from the FROG measurement (Error $27.10^{-4}$ on a $256 \times 256$ grid).

Figure 3 compares the input spectrum delivered by the frontend and the spectrum of the amplified pulses at maximum energy. The spectral bandwidth is well preserved throughout the amplification and compression optics. We observe a significant steepening on the long wavelength side of the spectrum at high energy due to gain saturation of the amplifiers that tends to amplify more the leading edge of the pulses, i.e. the long wavelengths, which shifts the maximum of the spectrum to $1035 \mathrm{~nm}$. The spectral bandwidth measured at maximum compressed energy is $\sim 8.5 \mathrm{~nm}$ and supports transform-limited pulses of $240 \mathrm{fs}$ duration (corresponding TBP 0.58).

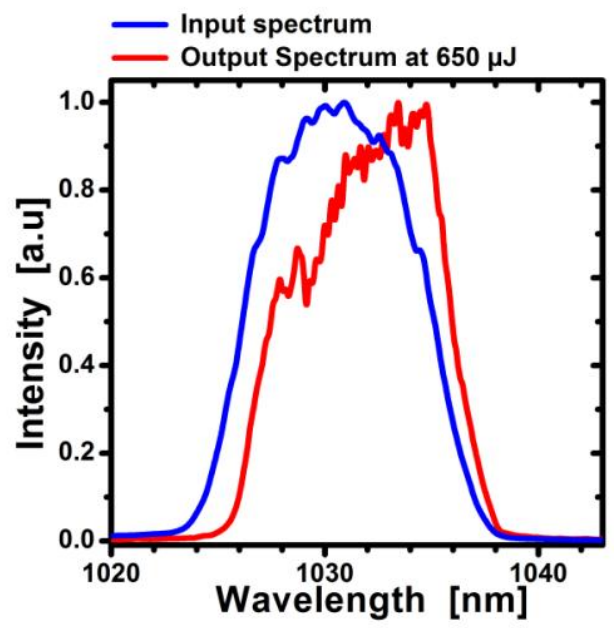

Figure 4: (Color online) Optical spectrum measured (blue) at the output of the frontend and (red) after compression at maximum energy.
Finally, we analyze the beam quality after compression. $\mathrm{M}^{2}$ measurements are systematically carried out and show no degradation of the beam quality with increasing combined average power with constant values of $\mathrm{M}^{2}{ }_{\mathrm{x}}=$ 1.25 and $\mathrm{M}_{\mathrm{y}}^{2}=1.15$ after the compressor.

In conclusion, we demonstrate a significant performance scaling of the passive coherent combining concept introduced in Ref. [5] in a moderately nonlinear FCPA. The use of state-of-the-art ytterbium-doped rod-type photonic crystal fibers permits to generate ultrashort pulses with $650 \mu \mathrm{J}$ of energy per pulse together with a measured duration of $300 \mathrm{fs}$ at a repetition rate of $92 \mathrm{kHz}$. The very good temporal quality of the recompressed pulses allows obtaining more than $2 \mathrm{GW}$ of peak power. At the higher repetition rate of $2 \mathrm{MHz}, 135 \mathrm{~W}$ of passively coherently combined average power (limited by the available pump average power) resulting in $105 \mathrm{~W}$ of compressed average power has been measured which is to our knowledge the highest average power ever reported from an ultrafast coherent combining system. All the performances reported in this Letter have been obtained with combination efficiencies over $91 \%$. Moreover, a careful preparation of the end-facets of the fibers together with a better thermo-mechanical management could help increasing this value. The authors strongly believe that this concept of amplification has the potential to generate more than $10 \mathrm{GW}$. This could be directly obtained with an additional post compression module base on a hollow core fiber or by increasing the stretching ratio to the level of Ref. [1]. The average power of these fiber systems could also be scaled to $1.5 \mathrm{~kW}$ by using adequate fiber designs such as those demonstrated in Ref. [9], with pulse duration well below $500 \mathrm{fs}$ in all cases.

\section{References}

[1] Tino Eidam, Jan Rothhardt, Fabian Stutzki, Florian Jansen, Steffen Hädrich, Henning Carstens, Cesar Jauregui, Jens Limpert, and Andreas Tünnermann, Opt. Express 19, 255-260 (2011)

[2] Enrico Seise, Arno Klenke, Jens Limpert, and Andreas Tünnermann, Opt. Express 18, 27827-27835 (2010)

[3] Arno Klenke, Enrico Seise, Stefan Demmler, Jan Rothhardt, Sven Breitkopf, Jens Limpert, and Andreas Tünnermann, Opt. Express 19, 24280-24285 (2011)

[4] T. Shay, V. Benham, J. T. Baker, A. D. Sanchez, D. Pilkington, and C. A. Lu, IEEE J. Sel. Top. Quantum Electron. 13, 480 (2007).

[5] L. Daniault, M. Hanna, L. Lombard, Y. Zaouter, E. Mottay, D. Goular, P. Bourdon, F. Druon, and P. Georges, Opt. Lett. 36, 621$623(2011)$

[6] Louis Daniault, Marc Hanna, Dimitris N. Papadopoulos, Yoann Zaouter, Eric Mottay, Frédéric Druon, and Patrick Georges, Opt. Lett. 36, 4023-4025 (2011)

[7] Y. Zaouter, J. Boullet, E. Mottay, and E. Cormier, Opt. Lett. 33, 1527-1529 (2008)

[8] F. Rös9]er, D. Schimpf, J. Rothhardt, T. Eidam, J. Limpert, A. Tünnermann, and F. Salin, Advanced Solid-State Photonics, OSA 2008 , paper WB22.

[9] Tino Eidam, Stefan Hanf, Enrico Seise, Thomas V. Andersen, Thomas Gabler, Christian Wirth, Thomas Schreiber, Jens Limpert, and Andreas Tünnermann, Opt. Lett. 35, 94-96 (2010) 


\section{References:}

[1] Tino Eidam, Jan Rothhardt, Fabian Stutzki, Florian Jansen, Steffen Hädrich, Henning Carstens, Cesar Jauregui, Jens Limpert, and Andreas Tünnermann, "Fiber chirped-pulse amplification system emitting $3.8 \mathrm{GW}$ peak power," Opt. Express 19, 255-260 (2011)

[2] Enrico Seise, Arno Klenke, Jens Limpert, and Andreas Tünnermann, "Coherent addition of fiber-amplified ultrashort laser pulses," Opt. Express 18, 27827-27835 (2010)

[3] Arno Klenke, Enrico Seise, Stefan Demmler, Jan Rothhardt, Sven Breitkopf, Jens Limpert, and Andreas Tünnermann, "Coherently-combined two channel femtosecond fiber CPA system producing $3 \mathrm{~mJ}$ pulse energy," Opt. Express 19, 2428024285 (2011)

[4] T. Shay, V. Benham, J. T. Baker, A. D. Sanchez, D. Pilkington, and C. A. Lu, IEEE J. Sel. Top. Quantum Electron. 13,480 (2007).

[5]L. Daniault, M. Hanna, L. Lombard, Y. Zaouter, E. Mottay, D. Goular, P. Bourdon, F. Druon, and P. Georges, "Coherent beam combining of two femtosecond fiber chirped-pulse amplifiers," Opt. Lett. 36, 621-623 (2011)

[6] Louis Daniault, Marc Hanna, Dimitris N. Papadopoulos, Yoann Zaouter, Eric Mottay, Frédéric Druon, and Patrick Georges, "Passive coherent beam combining of two femtosecond fiber chirped-pulse amplifiers," Opt. Lett. 36, 4023-4025 (2011)

[7] Y. Zaouter, J. Boullet, E. Mottay, and E. Cormier, "Transformlimited $100 \mathrm{\mu J}, 340 \mathrm{MW}$ pulses from a nonlinear-fiber chirpedpulse amplifier using a mismatched grating stretchercompressor," Opt. Lett. 33, 1527-1529 (2008)

[8] F. Röser, D. Schimpf, J. Rothhardt, T. Eidam, J. Limpert, A. Tünnermann, and F. Salin, "Gain Limitations and Consequences for Short Length Fiber Amplifiers," in Advanced Solid-State Photonics, OSA Technical Digest Series (CD) (Optical Society of America, 2008), paper WB22.

[9] Tino Eidam, Stefan Hanf, Enrico Seise, Thomas V. Andersen, Thomas Gabler, Christian Wirth, Thomas Schreiber, Jens Limpert, and Andreas Tünnermann, "Femtosecond fiber CPA system emitting $830 \mathrm{~W}$ average output power," Opt. Lett. 35, 9496 (2010) 